\title{
Anti-PD-1 elicits regression of undifferentiated pleomorphic sarcomas with UV-mutation signatures
}

To cite: Cheung LS, Chen L, Oke TF, et al. Anti-PD-1 elicits regression of undifferentiated pleomorphic sarcomas with UV-mutation signatures. Journal for ImmunoTherapy of Cancer 2021;9:e002345. doi:10.1136/ jitc-2021-002345

- Additional online supplemental material is published online only. To view, please visit the journal online (http://dx.doi.org/10.1136/jitc2021-002345).

Accepted 18 April 2021

Check for updates

(C) Author(s) (or their employer(s)) 2021. Re-use permitted under CC BY-NC. No commercial re-use. See rights and permissions. Published by BMJ.

For numbered affiliations see end of article.

Correspondence to

Dr Nicolas Llosa;

nllosa1@jhmi.edu

\section{ABSTRACT}

Undifferentiated pleomorphic sarcoma (UPS), an aggressive soft-tissue sarcoma of adults, has been characterized by low tumor mutational burden (TMB) and high copy number alterations. Clinical trials of programmed death-1 (PD-1) blockade in UPS have reported widely varying efficacy. We describe two patients with recurrent scalp UPS that experienced clinical benefit from PD-1 blockade. These tumors had high TMB with a UV-induced mutational pattern. Analysis of additional head and neck UPS cases identified five out of seven tumors with high TMB and an ultraviolet (UV) mutational signature. Head and neck UPS tumors also had increased programmed death-ligand 1 (PD-L1) expression and CD8+ T cell infiltration as compared with UPS tumors arising from other sites. In summary, we found that UPS tumors of the head and neck, but not elsewhere, have a PD$\mathrm{L} 1+$, T-cell-inflamed tumor microenvironment and high TMB, suggesting that these tumors represent a distinct genetic subgroup of UPS for which immune checkpoint inhibitor therapy might be effective.

\section{BACKGROUND}

Soft-tissue sarcomas (STS) comprise a heterogeneous group of cancers that arise from mesenchymal tissue and represent $\sim 1 \%$ of adult malignancies. Undifferentiated pleomorphic sarcomas (UPS) are typically deep-seated lesions that enlarge rapidly and painlessly, frequently located in the limbs followed by the trunk, while superficial lesions are rare. The pathogenesis is unknown, although some tumors occur in a previously irradiated field. ${ }^{1}$ Surgery plus radiotherapy remain the cornerstone of treatment of nonmetastatic tumors. Relapse is common, with a 5-year overall survival rate of $\sim 64 \%$, and prognosis for patients with metastatic disease is poor, with a median survival of $\sim 12$ months. $^{2}$
Treatment options for advanced UPS remain limited. Although PD-1 blockade has triggered tumor regressions in some patients, clinical trial results have been mixed. While Tawbi and colleagues reported a $40 \%(4 / 10)$ objective response rate to anti-PD-1, other trials have reported fewer responders or none at all, suggesting that a subset of UPS patients may respond to immune checkpoint blockade. ${ }^{3-5}$ Emerging evidence reveals an association between increased tumor-infiltrating immune cells and tumor regressions. ${ }^{6}$ Recent clinical trials have shown efficacy of PD-1 blockade in high tumor mutational burden (TMB) tumors, across many solid tumor types, leading to approval of the drug for treatment of high TMB tumors, regardless of tumor type. ${ }^{7}$ Additionally, $>1 \%$ intratumoral PD-L1 expression in other tumor types is often associated with antitumor response to anti-PD-1, however, UPS generally have low TMB and the majority do not express high PD-L1. ${ }^{8-11}$ Further studies to determine how UPS patients who respond to immunotherapy differ from non-responders are necessary for prospectively identifying patients who may benefit from checkpoint blockade and to understand what aspects of the tumor and tumor immune microenvironment can promote response in UPS patients. Here, we report two patients with relapsed and metastatic UPS of the scalp who were found to have unusually high TMB, but were microsatellite stable. Both patients were treated with checkpoint blockade and experienced clinical improvement. We analyzed the mutational signatures and tumor microenvironment of these and other UPS tumors to determine if UPS of 
the head and neck have distinct characteristics relevant to response to checkpoint blockade.

\section{METHODS \\ Patients and tumor samples}

Both patients' primary UPS tumors were not associated with previous radiation therapy. Tumor tissues and blood were collected at Johns Hopkins Hospital (Baltimore, Maryland, USA) from patients with UPS. All samples were obtained in accordance with the Health Insurance
Portability and Accountability Act. Immunohistochemical analysis was performed on samples from the two patients detailed in the case report and 36 unique patients with UPS obtained from the Johns Hopkins Hospital surgical pathology archives from 2005 to 2017. All radiationinduced UPS tumors were excluded. Further details regarding patient treatment and demographics are provided in online supplemental table 1 . Whole-exome sequencing (WES) data of head and neck UPS samples was obtained from the two case report patients, three
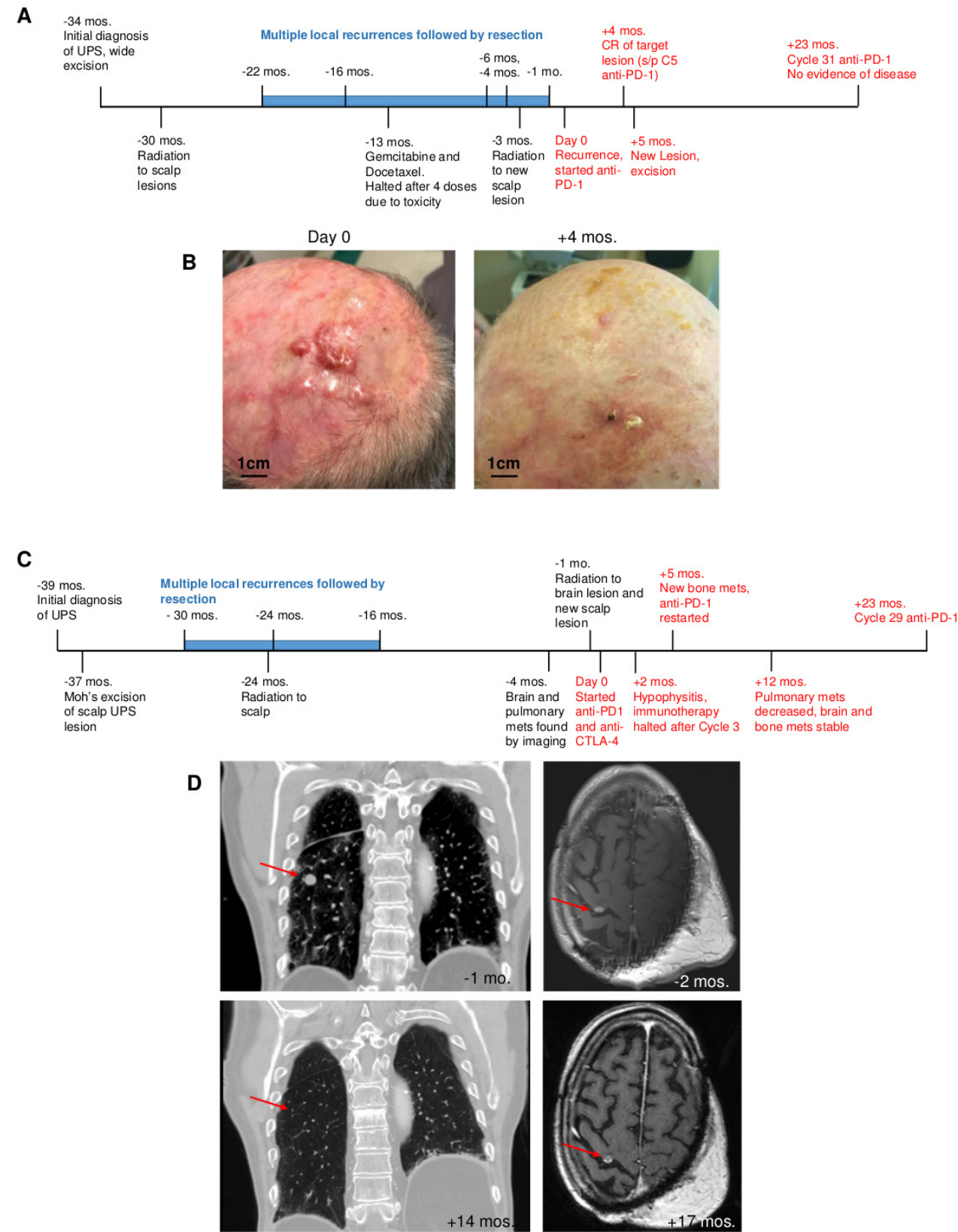

Figure 1 Clinical timeline and response to checkpoint blockade in two patients with ups of the scalp. Day 0 represents initiation of immunotherapy treatment. Events in red occurred while patients were treated with immunotherapy. (A) Clinical timeline of treatment for patient 1. (B) Images of patient 1 scalp lesions from before (left) and after achieving a CR of the target lesion 4 months after initiation of immunotherapy (right). (C) Clinical timeline of treatment for patient 2. (D) CT imaging of chest and MRI of brain from patient 2 before (top) and during (bottom) immunotherapy treatment exhibiting resolution of lung metastatic lesion and stabilization of brain metastatic mass. UPS, undifferentiated pleomorphic sarcoma.; CR, complete response. 
patients from the Johns Hopkins Hospital surgical pathology archives, one patient from the Cancer Genome Atlas (TCGA) and one patient from MSK-IMPACT clinical sequencing cohort(online supplemental table 2). ${ }^{12} 13$ Genomic data for 48 non-head and neck UPS samples were from TCGA.

\section{Immunohistochemistry}

Formalin-fixed paraffin-embedded tissue blocks from tumor specimens were annotated by a pathologist, cut into 5 um sections and mounted onto plus-charge glass slides. For each specimen, staining was performed according to standard protocol.

\section{RESULTS}

\section{Case presentation number 1}

A male in his late 60s with Fitzpatrick skin type II and a history of basal cell and cutaneous squamous cell carcinoma presented with a scalp lesion confirmed to be high grade UPS and was initially treated with surgical wide excision of the lesion, followed by radiation therapy. Over the next 18 months, the patient presented with multiple recurrences of scalp lesions, which were resected. He received gemcitabine and docetaxel, which was discontinued due to toxicities, and additional doses of radiation to his scalp but continued to develop new scalp lesions. Targeted DNA sequencing of his lesions revealed a microsatellite stable high TMB tumor. From the time of diagnosis, the patient underwent six total surgical resections and two cycles of radiation, but due to progression of his disease and high TMB of his tumor, he was enrolled on a phase 2 clinical trial with anti-PD-1 therapy for high TMB neoplasms (ClinicalTrials.gov number, NCT01876511). The largest scalp lesion measured $20 \mathrm{~mm}$ in diameter with several additional satellite lesions. After 4 months of anti-PD-1 treatment, the patient had a complete response (defined by Response Evaluation Criteria in Solid Tumors, RECIST V.1.1) of his dominant scalp lesion and satellite scalp lesions. ${ }^{14}$ One month later, a new scalp lesion emerged. However, given that the dominant scalp lesion was still undetectable and there was no evidence of metastatic disease, the new lesion was resected and the patient continued on anti-PD-1 after surgical recovery. The patient has now been treated with anti-PD-1 for 22 months and continues to have no evidence of local or distant disease for the past 16 months (figure 1A,B).

\section{Case presentation number 2}

A man in his late 70 s with Fitzpatrick skin type II and a history of cutaneous squamous cell carcinoma presented
A

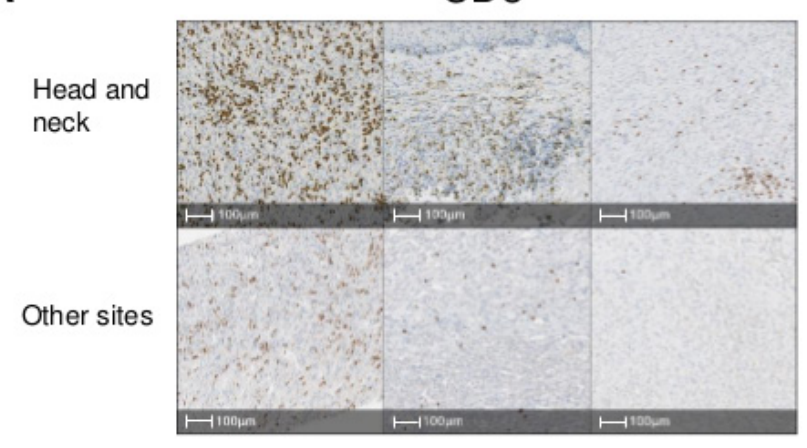

B

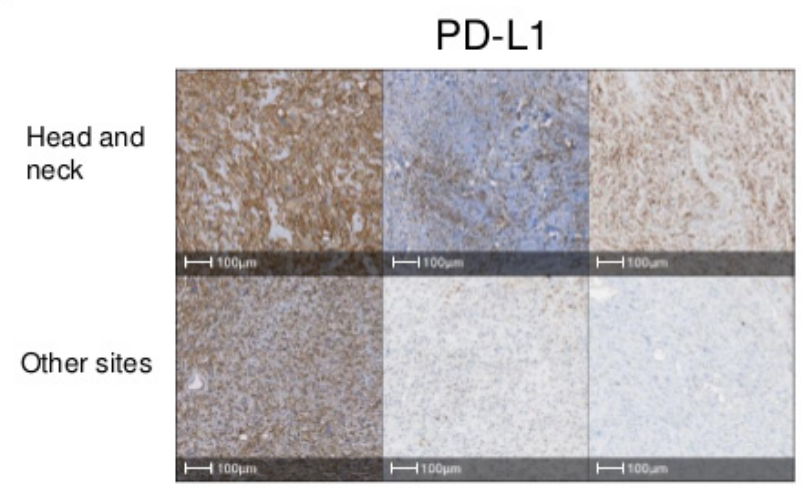

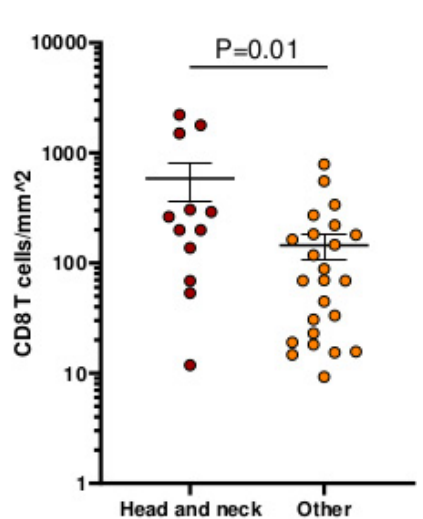

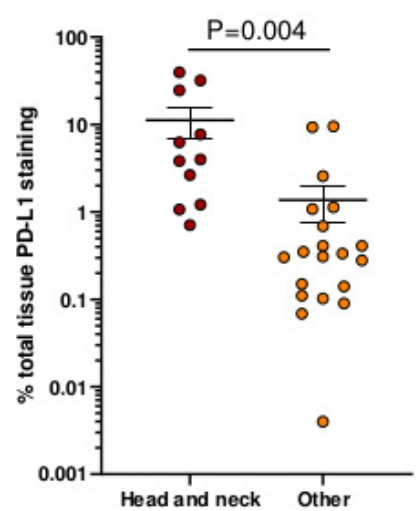

Figure 2 CD8+ Tcell infiltration and PD-L1 expression in UPS tumors of head and neck compared with other sites. (A) Representative images and quantitation of IHC staining of UPS tumors for CD8. The number of CD8+ Tcells $\mathrm{mm}^{2}$ of tumor tissue is shown. (B) Representative images and quantifation of IHC staining of ups tumors for PD-L1. Per cent PD-L1 expression of tumor tissue is shown. I bars show the SE. P values were calculated using a two-tailed t-test. UPS, undifferentiated pleomorphic sarcoma; IHC, immunohistochemistry. 
with a high grade UPS of his occipital scalp. Targeted DNA sequencing of the tumor found it to be microsatellite stable with a high TMB. He underwent wide excision but experienced disease relapse 7 months later requiring a radical resection, followed by adjuvant radiation therapy. Despite undergoing several surgical resections, the patient developed brain, osseous, and lung metastases. The patient received radiation to the brain lesion and to a new scalp lesion. One month later on follow-up CT, several lung nodules were noted to be increasing in size. The lung mass measured $13 \mathrm{~mm}$ and the brain lesion was $7 \mathrm{~mm}$ in diameter pretreatment. Immunotherapy with combination anti-PD-1 and anti-CTLA-4 was started. After 1 month of anti-PD-1, the patient had regression of lung nodules with stabilization of the brain lesion. At month 2 of anti-PD-1 and anti-CTLA-4, the patient developed hypophysitis requiring administration of replacement hydrocortisone therapy and immunotherapy was halted. Imaging demonstrated new osseous metastases, but pulmonary nodules continued to shrink and the brain lesion remained stable and anti-PD-1 monotherapy was restarted 3 months later. The patient has had complete resolution of pulmonary nodules, with stable brain and bone lesions for the last 18 months after 23 months of anti-PD-1 therapy (figure 1C,D).

\section{WES and mutational signature}

As targeted sequencing uses a panel of commonly mutated genes and can only provide an estimated TMB, WES was performed to determine the exact TMB for each tumor. High TMB is generally defined as $\geq 10$ mutations/ MB. ${ }^{15}$ TMB was 33 and 43 mutations/MB for the patients, respectively, while the average TMB of UPS tumors in the TCGA is 2.7 mutations/MB, indicating these scalp tumors were likely subjected to a different mutational process. To assess mutational signatures of these tumors, we compared the frequencies of single nucleotide variants to reference signatures in the COSMIC database. We found that for both tumors, COSMIC signature 7 was the dominant signature, with a prevalence of $\mathrm{C}>\mathrm{T}$ mutations in a dipyrimidine context, which is characteristic of UV-induced DNA damage and repair and is frequently found in melanoma (online supplemental figures 1A, 2A) ${ }^{16}$ This finding is interesting as a previous study characterizing genomics of STS identified only one out of 44 UPS tumors with high TMB due to a mutation in DNA mismatch repair machinery, rather than being due to UV exposure. $^{8}$

To determine whether UV damage is common in head and neck UPS tumors, we analyzed mutational signatures of UPS tumors in publically available databases as well as tumors from three patients who underwent surgical resection at Johns Hopkins Hospital. Of three head and neck UPS tumors resected from Johns Hopkins patients that we sequenced, one patient also had high TMB at 68 mutations/MB with a UV mutational signature, while the other two patients had less than 10 mutations/MB. Additionally, we found two patients with primary UPS tumors arising from the head and neck in the TCGA and MSKIMPACT databases and both tumors had high TMB (53 and 62 mutations/MB) with a UV mutational signature (online supplemental figures 2, 3, online supplemental table 2). ${ }^{12}{ }^{13}$ In the TCGA dataset, one non head and neck UPS tumor had high TMB, located at the thigh/ knee with a UV signature (data not shown), however, 26 other UPS tumors located on the leg were low TMB. In summary, of seven head and neck UPS tumors for which sequencing data was available, five tumors had high TMB due to UV exposure.

\section{T cell infiltration and PD-L1 expression in the tumor microenvironment}

CD8 + Tcell infiltration and PD-L1 expression have been found to correlate with anti-PD-1 response, independently of TMB. ${ }^{17}$ While UPS tumors generally have a paucity of T cells and low expression of PD-L1, tumors from patient 1 and 2 had high levels of infiltrating CD8 $+\mathrm{T}$ cells and expression of PD-L1 (online supplemental figure $1 \mathrm{~B}) .{ }^{11}$ We analyzed 36 additional UPS samples from the Johns Hopkins Hospital archive, comparing the levels of CD8 + T cells and PD-L1 expression in head and neck UPS tumors to those arising from other sites in the body (online supplemental table 1). Head and neck UPS tumors had increased CD8 + T cell infiltration and expression of PD-L1 versus UPS tumors from other sites of the body (figure 2A,B). High TMB did not predict the level of CD8+ T cells or PD-L1 in the tumor.

\section{DISCUSSION}

We report two patients with recurrent scalp UPS tumors with high TMB and UV mutational signatures who experienced clinical benefit with anti-PD-1 therapy. Together with analysis of additional head and neck UPS samples, our findings suggest that UPS tumors arising in this location may represent a distinct genetic subgroup of UPS with predominantly UV-induced mutations that confer susceptibility to checkpoint blockade. Both patients had developed frequent locally recurrent tumors, and in one case, metastatic lesions before starting immunotherapy. Additionally, patients were unable to continue with standard chemotherapy due to treatment failure or intolerable toxicities. On treatment with antiPD-1, both patients derived clinical benefit from checkpoint blockade. Further analysis of head and neck UPS tumors revealed additional cases with a UV mutational signature and a T-cell-inflamed immune signature as compared with UPS tumors arising from other sites of the body.

UPS tumors have been previously reported to respond to immunotherapy, however, the majority are not sensitive to anti-PD-1 treatment. ${ }^{3-5}$ In these trials, the location of the primary tumors (ie, head and neck vs elsewhere) were not reported. UPS generally has low TMB, and therefore, generates fewer tumor neoantigens, decreasing the likelihood of T cell recognition of the tumor. ${ }^{18}$ High TMB can occur due to environmental factors such as exposure to carcinogens or mutations in DNA repair pathways that allow for the accrual 
of errors during DNA replication. ${ }^{19}{ }^{20} \mathrm{UV}$ rays only penetrate the epidermis and dermis, causing DNA damage in tumors such as melanoma and squamous cell carcinoma. While most UPS tumors originate in the deep tissues, a subset of tumors arise in the dermis, making these tumors more likely to have UV-induced high TMB. ${ }^{21}$ Our data suggest that UV damage can cause high TMB in UPS tumors of the head and neck, due to increased sun exposure of these sites, making these tumors more likely to be sensitive to checkpoint blockade than tumors arising from other sites. Of seven head and neck UPS tumors for which we obtained WES data, five tumors had high TMB with a UV signature, suggesting this may be a frequent phenomenon. Additionally, 1 of 26 leg UPS tumors also had high TMB, indicating that although non-head and neck tumors are less frequently high TMB, some UPS tumors arising in other sun-exposed sites may also be amenable to checkpoint blockade. Steele et al have also identified a subset of high TMB UPS tumors due to DNA repair pathway defects associated with IFN gamma response pathway enrichment, suggestive of increased immune infiltration, which mirrors our observations in UPS tumors with UV mutational signatures. ${ }^{20}$

Previously, studies of UPS immune infiltrates have shown low levels of CD8+ T cells and PD-L1 expression, despite being more inflamed when compared with other STS. ${ }^{1022}$ We found that head and neck UPS tumors had increased CD8+ Tcell infiltration and intratumoral PD-L1 expression as compared with UPS tumors located in other sites. This T-cell-inflamed phenotype could indicate a pre-existing anti-tumor immune response that has subsequently become exhausted, making these tumors more likely to respond to anti-PD-1 therapy. ${ }^{23}$

In conclusion, we describe two patients with high TMB scalp UPS tumors who experienced an anti-tumor immune response to checkpoint blockade. Head and neck UPS showed a higher frequency of UV-induced high TMB and a T-cell inflamed phenotype compared with UPS arising from other sites. Taken together, these findings support the screening of head and neck UPS tumors for high TMB and consideration for treatment with PD-1 blockade.

\author{
Author affiliations \\ ${ }^{1}$ Johns Hopkins Bloomberg Kimmel Institute for Cancer Immunotherapy, Baltimore, \\ Maryland, USA \\ ${ }^{2}$ Department of Oncology, Johns Hopkins Medicine Sidney Kimmel Comprehensive \\ Cancer Center, Baltimore, Maryland, USA \\ ${ }^{3}$ NextCure Inc, Beltsville, Maryland, USA \\ ${ }^{4}$ Department of Pathology, Johns Hopkins University School of Medicine, Baltimore, \\ Maryland, USA \\ ${ }^{5}$ Division of Solid Tumor Oncology, Memorial Sloan Kettering Cancer Center, New \\ York, New York, USA
}

Contributors LSC and NL conceptualized and designed the study. LSC, LC, and TBS analyzed data. LSC, LC, TFO, JT and HK collected clinical data. LSC, LC and NL interpreted data. LAD, EL, J-WS, JT, RA, DMP, DTL and CFM provided insight and assisted in interpretation of data. DTL, KB and CFM treated the cases. LSC and NL wrote the manuscript. NL supervised the study. All authors reviewed and edited manuscript.

Funding This work was supported by Johns Hopkins Hospital, Bloomberg-Kimmel Institute for Immunotherapy; Bloomberg Philanthropies, BMS II-ON, MERCK Pharmaceuticals, Giant Food, and a Stand Up to Cancer Colorectal Cancer Dream
Team Translational Research Grant. Stand Up to Cancer is a program of the Entertainment Industry Foundation administered by the American Association for Cancer Research.

Competing interests $L C$ is employed by Janssen Research and Development at the time of this submission. LAD is a member of the board of directors of Personal Genome Diagnostics (PGDx) and Jounce Therapeutics. He is a paid consultant to PGDx, 4Paws (PetDx), Innovatus CP and Neophore. He is an uncompensated consultant for Merck but has received research support for clinical trials from Merck. LAD is an inventor of multiple licensed patents related to technology for circulating tumor DNA analyses and mismatch repair deficiency for diagnosis and therapy from Johns Hopkins University. Some of these licenses and relationships are associated with equity or royalty payments directly to Johns Hopkins and LAD. He holds equity in PGDx, Jounce Therapeutics, Thrive Earlier Detection and Neophore. His spouse holds equity in Amgen. The terms of all these arrangements are being managed by Johns Hopkins and Memorial Sloan Kettering in accordance with their conflict of interest policies. EJL has received institutional research grant funding from Bristol-Myers Squibb, Merck, and Regeneron. EJL is a consultant for Array BioPharma, Bristol-Myers Squibb, EMD Serono, MacroGenics, Novartis, Merck, Regeneron, Sanofi Genzyme. JMT serves on advisory boards for BMS, Merck, Akoya Biosciences, Astra Zeneca and has received research funding from BMS, Akoya Biosciences. RAA serves on advisory boards for Merck, Bristol Myers Squibb and FLX bio and has received research funding from Merck, Bristol Myers Squibb, and FLX bio. DMP serves on advisory boards for Merck, Bristol Myers Squibb and Compugen and has received research funding from Bristol Myers Squibb and Compugen. DTL serves on advisory boards for Merck and Bristol Myers Squibb and has received research funding from Merck, Bristol Myers Squibb, Aduro Biotech, Curegenix, Medivir, and Nouscom. She has received speaking honoraria from Merck and is an inventor of licensed intellectual property related to technology for mismatch repair deficiency for diagnosis and therapy (W02016077553A1) from Johns Hopkins University. The terms of these arrangements are being managed by Johns Hopkins. CFM was a paid consultant for Bayer and has received speakers bureau honoraria from Novartis. NJL has received funding support from Bristol Myers Squibb.

\section{Patient consent for publication Obtained.}

Ethics approval This study was conducted in accordance with the ethical principles stated in the Belmont Report and the US Common Rule. It was approved by the Johns Hopkins Institutional Review Board and all samples were obtained in accordance with the Health Insurance and Accountability Act. This study was conducted under an Institutional Review Board approved protocol with a waiver of consent for archived tissues and patients gave written informed consent for prospective tumor collection.

Provenance and peer review Not commissioned; externally peer reviewed.

Supplemental material This content has been supplied by the author(s). It has not been vetted by BMJ Publishing Group Limited (BMJ) and may not have been peer-reviewed. Any opinions or recommendations discussed are solely those of the author(s) and are not endorsed by BMJ. BMJ disclaims all liability and responsibility arising from any reliance placed on the content. Where the content includes any translated material, BMJ does not warrant the accuracy and reliability of the translations (including but not limited to local regulations, clinical guidelines, terminology, drug names and drug dosages), and is not responsible for any error and/or omissions arising from translation and adaptation or otherwise.

Open access This is an open access article distributed in accordance with the Creative Commons Attribution Non Commercial (CC BY-NC 4.0) license, which permits others to distribute, remix, adapt, build upon this work non-commercially, and license their derivative works on different terms, provided the original work is properly cited, appropriate credit is given, any changes made indicated, and the use is non-commercial. See http://creativecommons.org/licenses/by-nc/4.0/.

\section{ORCID iDs}

Evan Lipson http://orcid.org/0000-0003-2976-0911

John-WIlliam Sidhom http://orcid.org/0000-0002-5575-0285

Nicolas Llosa http://orcid.org/0000-0001-7047-0858

\section{REFERENCES}

1 Widemann BC, Italiano A. Biology and management of undifferentiated pleomorphic sarcoma, myxofibrosarcoma, and malignant peripheral nerve sheath tumors: state of the art and perspectives. J Clin Oncol 2018;36:160-7. 
2 Italiano A, Mathoulin-Pelissier S, Cesne AL, et al. Trends in survival for patients with metastatic soft-tissue sarcoma. Cancer 2011;117:1049-54

3 Tawbi HA, Burgess M, Bolejack V, et al. Pembrolizumab in advanced soft-tissue sarcoma and bone sarcoma (SARC028): a multicentre, two-cohort, single-arm, open-label, phase 2 trial. Lancet Oncol 2017;18:1493-501.

4 Toulmonde M, Penel N, Adam J, et al. Use of PD-1 targeting, macrophage infiltration, and IDO pathway activation in sarcomas: a phase 2 clinical trial. JAMA Oncol 2018;4:93-7.

5 D'Angelo SP, Mahoney MR, Van Tine BA, et al. Nivolumab with or without ipilimumab treatment for metastatic sarcoma (alliance A091401): two open-label, non-comparative, randomised, phase 2 trials. Lancet Oncol 2018;19:416-26.

6 Petitprez F, de Reyniès A, Keung EZ, et al. B cells are associated with survival and immunotherapy response in sarcoma. Nature 2020;577:556-60.

7 Marabelle A, Fakih MG, Lopez J, et al. Association of tumour mutational burden with outcomes in patients with select advanced solid tumours treated with pembrolizumab in KEYNOTE-158. Ann Oncol 2019;30:v477-8.

8 Abeshouse A, Adebamowo C, Adebamowo SN. Comprehensive and integrated genomic characterization of adult soft tissue sarcomas. Cell 2017;171:950-65.

9 Borghaei H, Paz-Ares L, Horn L, et al. Nivolumab versus docetaxel in advanced nonsquamous non-small-cell lung cancer. $N$ Engl J Med 2015;373:1627-39.

10 Park HK, Kim M, Sung M, et al. Status of programmed death-ligand 1 expression in sarcomas. J Trans/ Med 2018;16:1-11.

11 Boxberg M, Steiger K, Lenze U, et al. Pd-L1 and PD-1 and characterization of tumor-infiltrating lymphocytes in high grade sarcomas of soft tissue - prognostic implications and rationale for immunotherapy. Oncoimmunology 2018;7:e1389366-11.

12 Gao J, Aksoy BA, Dogrusoz U, et al. Integrative analysis of complex cancer genomics and clinical profiles using the cBioPortal. Sci Signal 2013;6:pl1-20.
13 Zehir A, Benayed R, Shah RH, et al. Mutational landscape of metastatic cancer revealed from prospective clinical sequencing of 10,000 patients. Nat Med 2017;23:703-13.

14 Eisenhauer EA, Therasse P, Bogaerts J, et al. New response evaluation criteria in solid tumours: revised RECIST guideline (version 1.1). Eur J Cancer 2009;45:228-47.

15 Hellmann MD, Ciuleanu T-E, Pluzanski A, et al. Nivolumab plus ipilimumab in lung cancer with a high tumor mutational burden. $N$ Engl J Med 2018;378:2093-104.

16 Tate JG, Bamford S, Jubb HC, et al. Cosmic: the Catalogue of somatic mutations in cancer. Nucleic Acids Res 2019;47:D941-7.

17 Yarchoan M, Albacker LA, Hopkins AC, et al. Pd-L1 expression and tumor mutational burden are independent biomarkers in most cancers. JCl Insight 2019;4. doi:10.1172/jci.insight.126908. [Epub ahead of print: 21 Mar 2019].

18 Rizvi NA, Hellmann MD, Snyder A, et al. Cancer immunology. mutational landscape determines sensitivity to PD-1 blockade in non-small cell lung cancer. Science 2015;348:124-8.

19 Chalmers ZR, Connelly CF, Fabrizio D, et al. Analysis of 100,000 human cancer genomes reveals the landscape of tumor mutational burden. Genome Med 2017;9:1-14.

20 Steele CD, Tarabichi M, Oukrif D, et al. Undifferentiated sarcomas develop through distinct evolutionary pathways. Cancer Cell 2019;35:441-56.

21 D'Orazio J, Jarrett S, Amaro-Ortiz A, et al. Uv radiation and the skin. Int J Mol Sci 2013;14:12222-48.

22 Keung EZ, Tsai J-W, Ali AM, et al. Analysis of the immune infiltrate in undifferentiated pleomorphic sarcoma of the extremity and trunk in response to radiotherapy: rationale for combination neoadjuvant immune checkpoint inhibition and radiotherapy. Oncoimmunology 2018;7:e1385689.

23 Trujillo JA, Sweis RF, Bao R, et al. T Cell-Inflamed versus non-T CellInflamed tumors: a conceptual framework for cancer immunotherapy drug development and combination therapy selection. Cancer Immunol Res 2018;6:990-1000. 